\title{
Development of Underwater Laser Scaling Adapter
}

\author{
Kaspars Bluss, Daugavpils University
}

\begin{abstract}
In this paper the developed laser scaling adapter is presented. The scaling adapter is equipped with a twin laser unit where the two parallel laser beams are projected onto any target giving an exact indication of scale. The body of the laser scaling adapter is made of Teflon, the density of which is approximately two times the water density. The development involved multiple challenges - numerical hydrodynamic calculations for choosing an appropriate shape which would reduce the effects of turbulence, an accurate sealing of the power supply and the laser diodes, and others. The precision is estimated by the partial derivation method. Both experimental and theoretical data conclude the overall precision error to be in the $1 \%$ margin. This paper presents the development steps of such an underwater laser scaling adapter for a remotely operated vehicle (ROV).
\end{abstract}

Keywords - laser scaling, remotely operated vehicle (ROV), seabed inspection

\section{INTRODUCTION}

One of the main challenges nowadays is the management of lakes in which changes take place due to both human involvement [1] and natural causes. Underwater seabed inspection is one of the most important tools for the evaluation of the ecosystem condition and executing a wide variety of inspection projects [2-5]. Thus continuous effort has been devoted for the development of devices and techniques which could ensure a fast and precise characterization of underwater objects and the seabed. In this contribution we have developed an underwater laser scaling adapter which can be mounted to a remotely operated vehicle (ROV). A ROV is a platform usually used for underwater investigations and can be equipped with multiple probes and gauges and can be used for seabed inspection [6-9]. The main components of the laser scaling device are the two laser diodes which are aligned so that their emitted laser beams are parallel. These laser beams are then projected on the subject under investigation and as the light is scattered or reflected it is detected by camera on ROV. In case one knows the actual distance between the laser beams, then from the projected spots one can actually measure the distance or the size of the object if one accounts for the effect of perspective. This means that the further the object under investigation is, the closer the points will seem to be. The distance and the seeming distance of the points are related with simple trigonometric equations.

The aim of this particular paper is to show the steps of developing a laser scaling device. First, fluid flow modeling must be performed for finding the optimal curvature of the edges of laser scaling device. Optimal conditions include less turbulence and smaller overall pressure on the faces of the device. The second step includes manufacturing of the body of the device according to assumptions made in previous step. Third, implementation and adjustment of the laser diodes is necessary. All of the components must be hermetically sealed. Afterwards, by performing some simple experiments, the system error can be obtained.

\section{NUMERICAL CALCULATIONS AND EXPERIMENTAL DEVELOPMENT}

Before the experimental development of the laser scaling device hydrodynamic, calculations were performed using commercially available software Comsol. The Comsol Laminar flow physics module was chosen for calculating fluid flows. The aim of the calculations was to estimate the optimal size and form of a laser scaling device that would have reduced hydrodynamic resistance (pressure on the boundaries) and turbulence at the boundaries of the laser scaling device. In Fig. 1 pressure distribution and fluid flow streamlines are shown. In the model pressure was applied to the lower boundary of the system. Upper boundary pressure was set to zero, pressure at all the other boundaries was also set to zero. The pressure difference causes the liquid phase (water) to flow around the scaling device. To reduce the effect of turbulence, the pressure was chosen small enough to have small water speeds. The maximum speed of water flow in this particular case was approximately $1.79 \mathrm{~mm} / \mathrm{s}$.

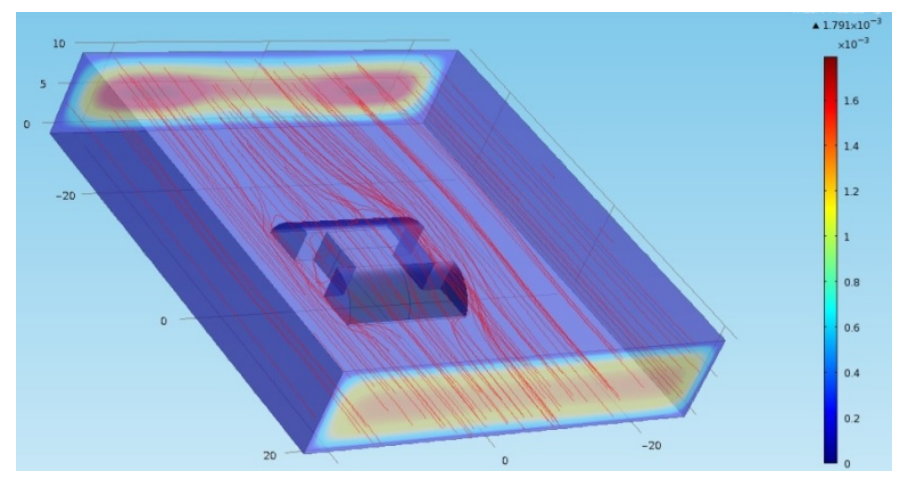

Fig. 1. Pressure distribution and fluid flow streamlines in a laminar fluid flow around the laser scaling device

Curving the corners of the device boundaries reduced the calculation time. Moreover, it is assumed that a problem with a more stable fluid flow without turbulence is calculated faster. Thus the model with smoothed edges of the adapter causes less turbulence and dissipation of energy. From this point of view smoothed edges are desirable for the device. Moreover, the overall pressure on the boundary reduced significantly if the edges are curved.

After theoretical calculations, drawings of the laser scaling device were made. The body of the laser scaling is made of Teflon. The only material considerations are the following material should be with higher density than that of water, material should be hard enough for threading. The Teflon 
adapter in process of development is shown in Fig. 2. In the picture one can see six smaller holes through which the adapter shall be fixed using regular bolts. In front of adapter two wider holes can be seen. These are meant for laser diodes on the one side of laser scaling device and Plexiglas shields on the other. The bottom part of the adapter was carefully polished. This must be done to have a good compatibility and thus airtight conditions between the adapter and the ROV.

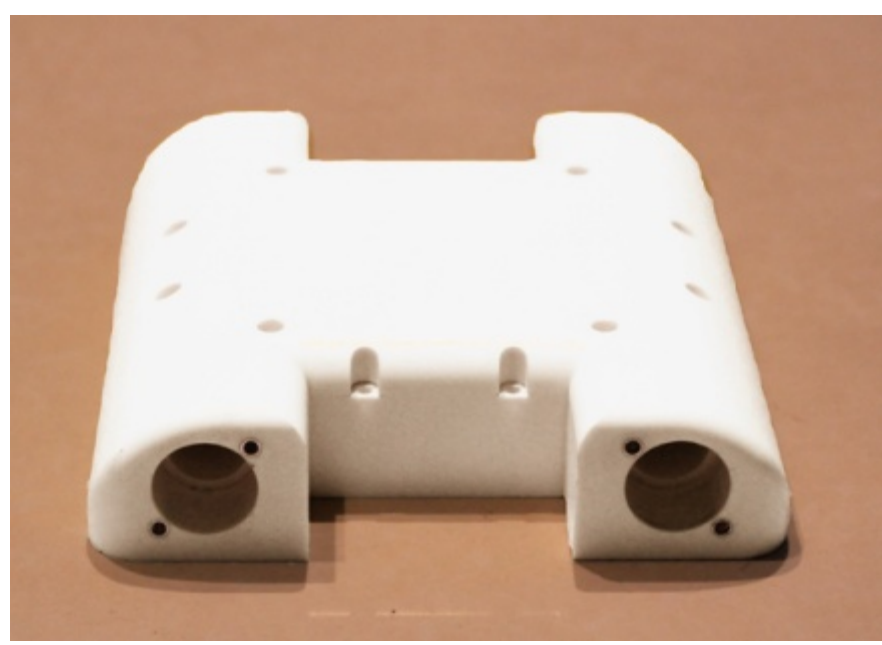

Fig. 2. Teflon adapter in process of development

Two $650 \mathrm{~nm}$ laser diodes which saturate at $12 \mathrm{~V}$ and approximately $35 \mathrm{~mA}$ were used as a light source. The considerations here are the low price of laser diodes. Laser diodes for shorter wavelengths are considerably more expensive than the ones for longer wavelengths. As the dispersion can vary with the medium this should be a secondary parameter to consider. The laser diodes are fed by the ROV.

The next important step is the adjustment of the laser diodes. The laser diodes are hermetically sealed and implemented in the adapter holes, which can be seen in Fig. 2. Screws and a rubber distancer are used to fix the laser diodes. The rubber distancer has a significant influence on the optimization of laser diode beam direction. Using the screws the laser beams are adjusted in several iterations. The distance $d$ between the beams as a function of distance $D$ from the adapter is measured. If the beams are parallel, then the distance $d$ should remain constant. The iterations measurement and screw adjustment - are performed until satisfactory conditions of the precision are met. The experimental data are shown in Fig. 3 - 5. To simplify the approximation process the beam separation length difference $\Delta d$ was registered as a function of distance from adapter $D$. In such case the tangent of approximated data should trend towards zero. As can be seen from Fig. 3 - 5, the tangent was decreased from $0.438 \mathrm{~mm} / \mathrm{m}$ for data $d_{3}$ obtained from laser diodes without optimization to $0.027 \mathrm{~mm} / \mathrm{m}$ for optimized data $d_{\text {opt }}$. The optimized value means that in hundred meters the laser beams will be additional $2.7 \mathrm{~mm}$ apart from each other comparing to the distance of $11 \mathrm{~mm}$ at the adapter. Assuming that the measurements will be performed in a medium with high turbidity measurements at a distance of $100 \mathrm{~m}$ would not be possible due to the fact that the laser light will certainly be absorbed within double the path length. The tangent value of the data will be used to calculate the overall measurement error of the device.

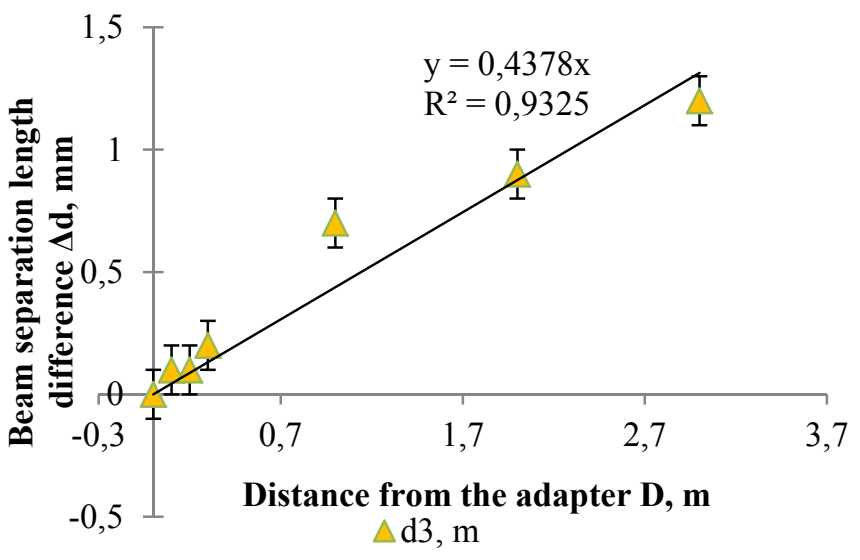

Fig. 3. Laser scaling device two beam separation length difference $\Delta d$ as a function of the distance from the adapter $D$ after first laser setup

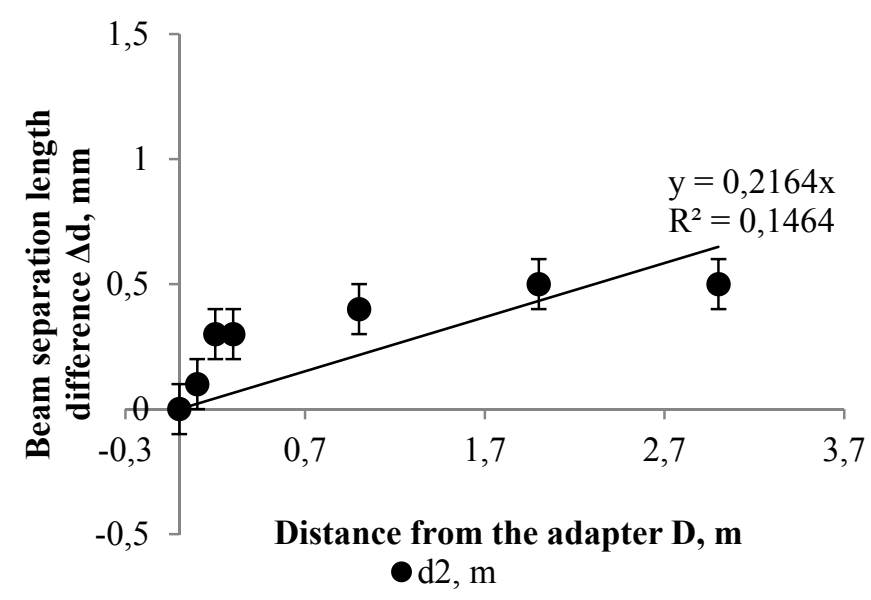

Fig. 4. Laser scaling device two beam separation length difference $\Delta d$ as a function of the distance from the adapter $D$ after first iteration step

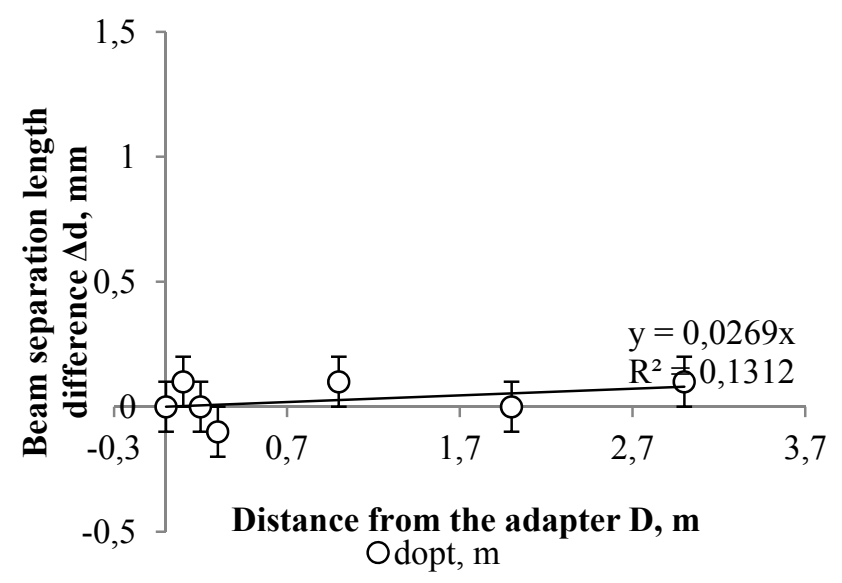

Fig. 5. Laser scaling device two beam separation length difference $\Delta d$ as a function of the distance from the adapter $D$ after second iteration step 


\section{RESULTS AND DISCUSSIONS}

Estimation of device measurement error was based on analysis of view angle in terms of perspective. See Fig. 6 for graphical interpretation.

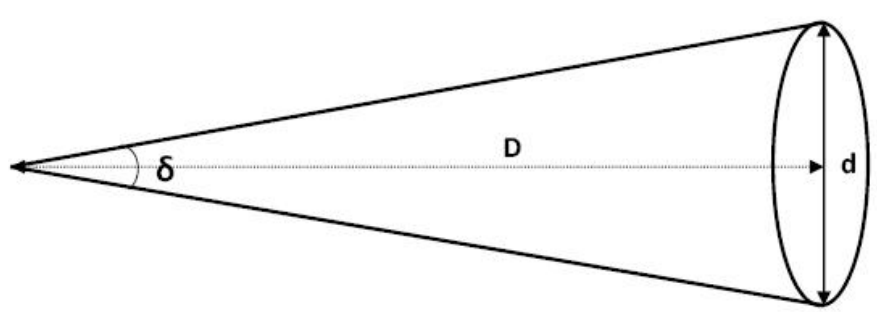

Fig. 6. View angle $\delta$ at object with size $\mathrm{d}$ at distance $D$

From Figure 6 it is obvious that

$$
\tan \delta=\frac{d}{D}
$$

Thus if object size $d$ is known than the distance can be estimated from

$$
D=\frac{d}{\tan \delta}=d \cdot \cot \delta
$$

Here the distance is a function of viewing angle, thus the viewing angle caused error can be calculated by

$$
\frac{\partial D}{\partial \delta}=\frac{d}{\left(-\csc ^{2} \delta\right)}=-d \cdot \sin ^{2} \delta .
$$

For calculation of relative error in percentage one must divide (3) by (2) thus obtaining

$$
\left|\frac{\partial D}{D}\right|=\frac{d \cdot \sin ^{2} \delta}{d \cdot \cot \delta} \partial \delta=\frac{\sin ^{3} \delta}{\cos \delta} \partial \delta .
$$

As can be seen from equation (4), the overall distance error does not hold the term $d$ which is the object size. The overall distance error holds only the viewing angle $\delta$ which can be estimated using the camera. The derived equation (4) can now be used to estimate the overall distance measurement error. First we assume that the view angle $\delta$ error (determined by experiment) $\partial \delta$ is small enough that

$$
\partial \delta=\tan k \approx k .
$$

The next task is to estimate the view angle $\delta$ range. The laser beams are $0.11 \mathrm{~m}$ apart which is used as the maximal estimation value. At a distance of one meter object with the size of $0.11 \mathrm{~m}$ will have view angle of $6.31^{\circ}$. Thus, the view angle range in a measurement will not exceed $40^{\circ}$. The relative error of distance as a function of view angle is shown in Fig. 7. From Fig. 7 one can see that the error does not exceed the $1 \%$ range.

Then installed laser scaling adapter can be seen in Figures 8 to 12 .

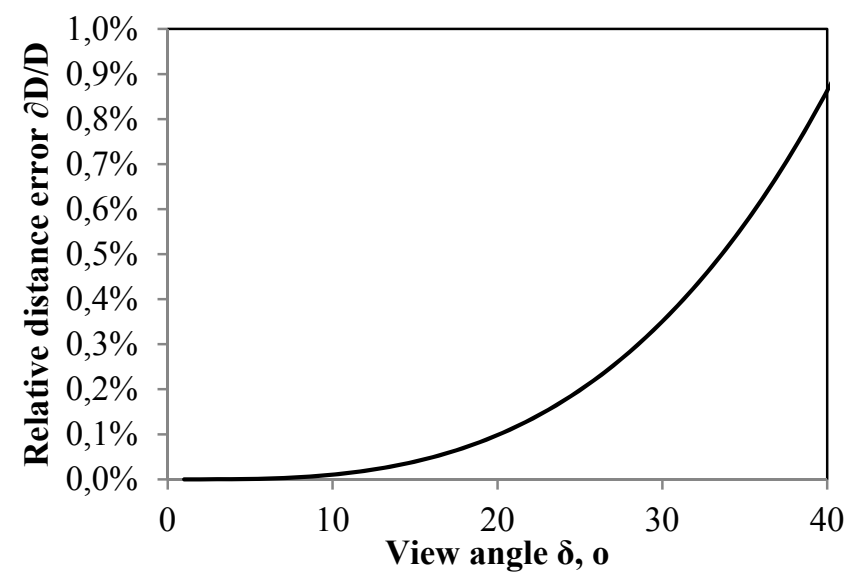

Fig. 7. Relative distance error $\partial D / D$ as a function of view angle $\delta$

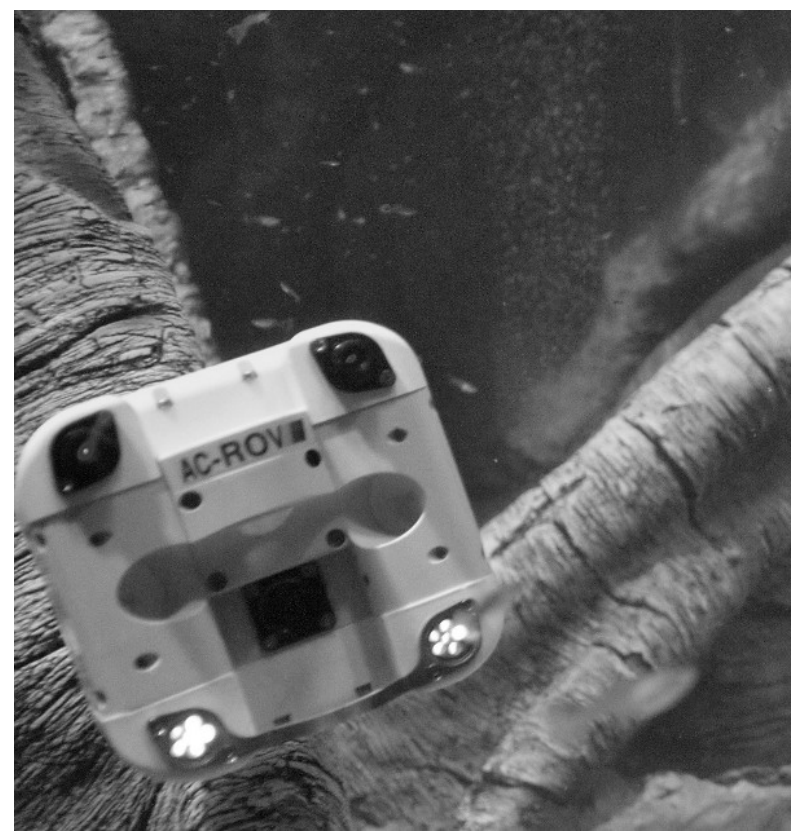

Fig. 8. Laser scaling adapter installed on a ROV - front view (1)

In Fig. 9 the laser diodes which project the points on the object under investigation are shown. The ROV manufactured by AC-CESS is equipped with propellers which allow one to control the position of the ROV. It is also equipped with LED light sources for a clear view of the seabed.

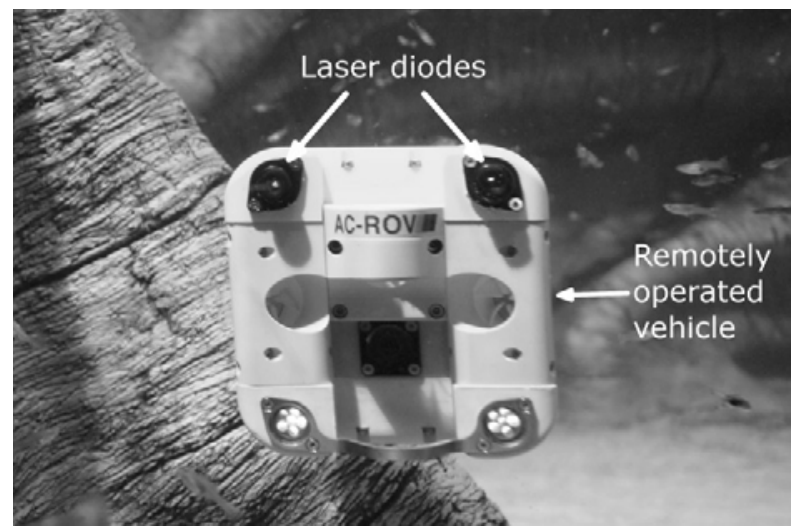

Fig. 9. Laser scaling adapter installed on ROV - front view (2) 


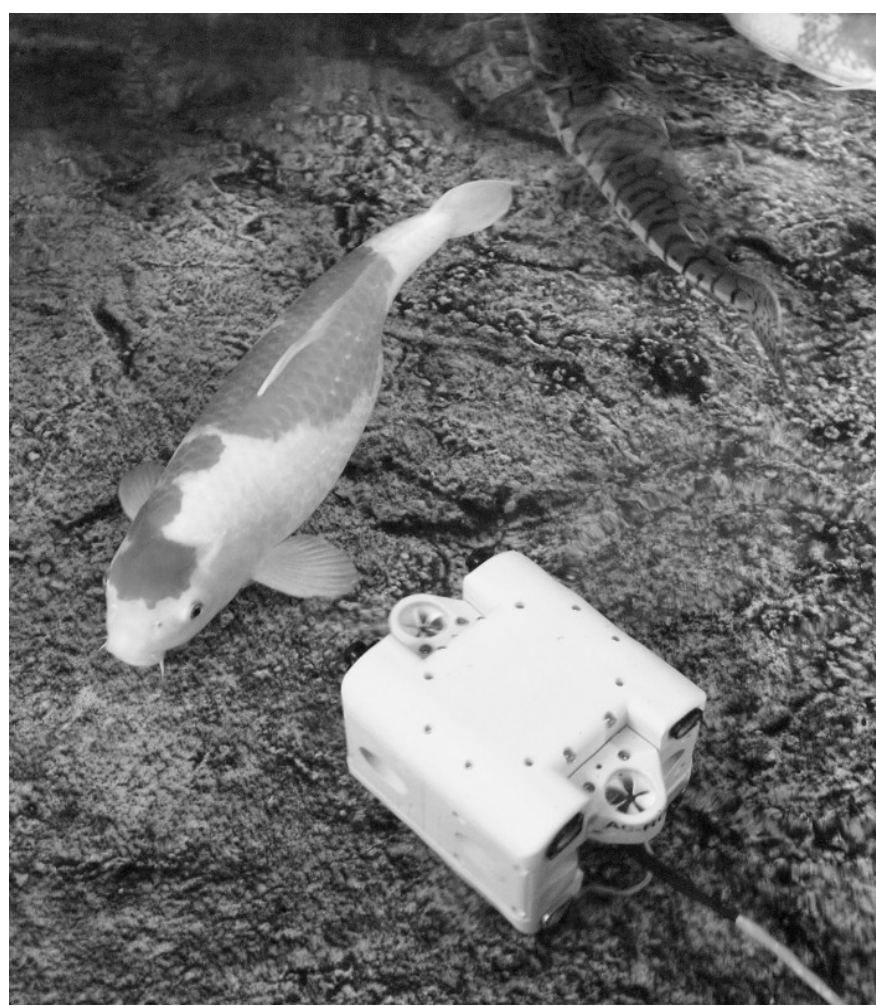

Fig. 10. Laser scaling adapter installed on ROV - top view (2)

In Fig. 11 one can see the laser diode projected points on the object. The distance from these points is determined by the distance between the diodes. By fixing the distance between the points using the camera installed in the ROV, one can actually determine the distance of the object from the ROV and the size of the object under investigation. The built-in camera is shown in Fig. 11 of this article. As can be seen from Fig. 12, the camera is built into the middle of the ROV.

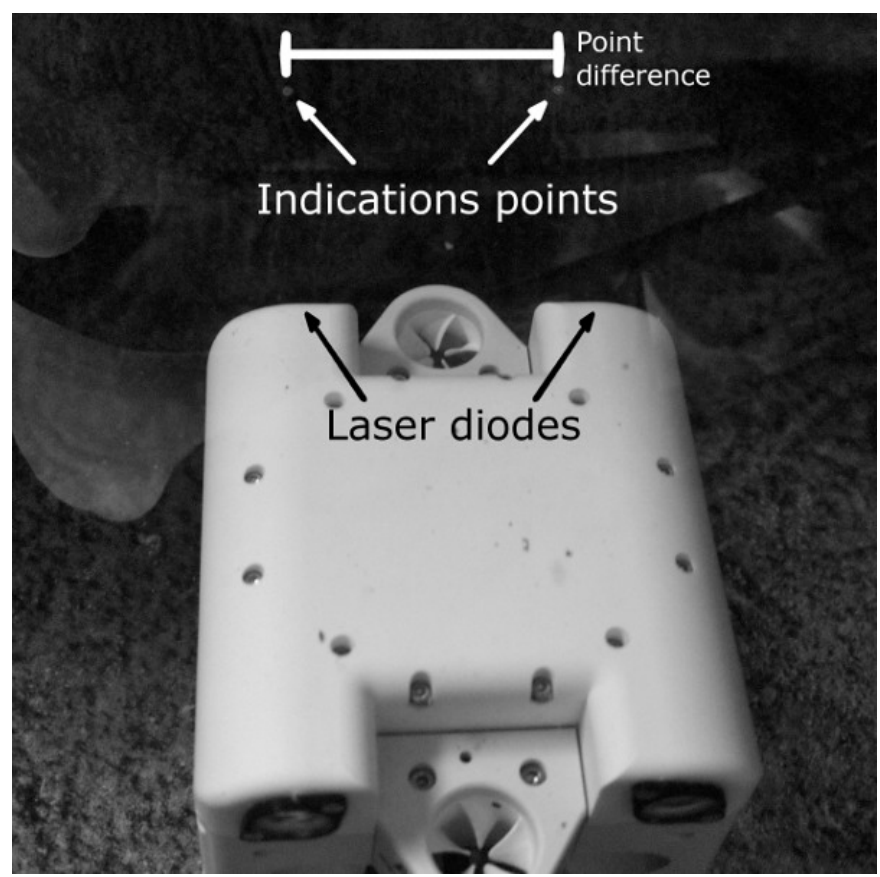

Fig. 11. Relative distance error $\partial D / D$ as a function of view angle $\delta$

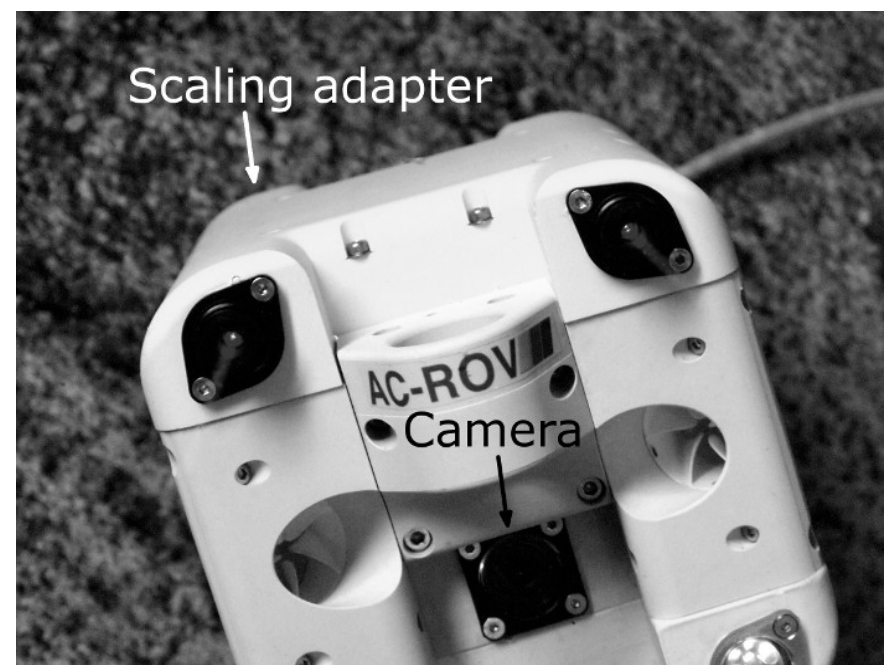

Fig. 12. Laser scaling adapter installed on ROV - front view (3)

\section{CONCLUSIONS}

The main steps for the development of a laser scaling adapter for a ROV are shown in this research. These include theoretical modeling fluid flows around the adapter for the aim of finding an optimal device shape, afterwards, creation of adapter body from Teflon, and implementation of laser diodes in the adapter. Last but not least, hermetical sealing of adapter must be ensured to avoid destroying the electronics after the adapter is tested in the water. It has also been shown that the developed laser adapter has the distance estimation precision of much less than 1 percent. The error value was estimated with the combination of values obtained in an independent experiment and derived equation.

\section{ACKNOWLEDGEMENTS}

This research has been supported by the European Social Fund (ESF) project "Formation of interdisciplinary research group for securing the sustainability of salmonid lakes in Latvia" Agreement No. 2009/0214/1DP/1.1.1.2.0/09/ APIA/VIAA/089

\section{REFERENCES}

1. Cooper K., Boyd S., Eggleton J., Limpenny D., Rees H., Vanstaen $\mathbf{K} .$, Recovery of the seabed following marine aggregate dredging on the Hastings Shingle Bank off the southeast coast of England, Estuarine, Coastal and Shelf Science 75, 2007, p. 547-558

2. Bruno F., Bianco G., Muzzupappa M., Barone S., Razionale A.V. Experimentation of structured light and stereo vision for underwater $3 D$ reconstruction, ISPRS Journal of Photogrammetry and Remote Sensing 66,2011 , p. $508-518$

3. Coggan R., Diesing M., The seabed habitats of the central English Channel: A generation on from Holme and Cabioch, how do their interpretations match-up to modern mapping techniques?, Continental Shelf Research 31, 2011, p. S132-S150

4. Jones, G.E., Cumming, R.E. \& Hunt, P.R. 2005. The Use of Remote Sensing Techniques for the Location and Investigation of Heritage Wreck Sites, Chapter in Maritime Heritage and Modern Ports, Ed. Marcet I Barbe, R., Brebbia, C.A. \& Olivella, J. WIT Press, Southampton. ISBN 1-84564-010-1.

5. Jones, G.E. \& Glegg, G.E. 2004. Effective Use of Geophysical Sensors for Marine Environmental Assessment and Habitat Mapping, Coastal EnvironmentV, incorp. Oil Spill Studies (Ed. C.A. Brebbia, et. al.), WIT Press, Southampton, ISBN 1-85312-710-8. 
$2012 / 10$

6. J. Crook, Deep-sea technology, Eng. \& Technol. -- 23 January 2010 -Volume 5, Issue 1, p.36-39

7. J.E. Stoll, Robots working beneath the sea, Surveyor New York, Volume 19, Issue 4, November 1985, Pages 2-7

8. T. Inoue, H. Osawa, H. Yoshida, J. Tahara, S. Ishibashi, K. Ito, Y. Watanabe, T. Sawa, T. Hyakudome, Sea trial results of ROV "ABISMO" for deep sea inspection and sampling, Proceedings of the International Conference on Offshore Mechanics and Arctic Engineering - OMAE 6, pp. 453-460

9. D. Morgan, Rising of the underwater challenge, Offshore Engineer 33 (8), pp. 82

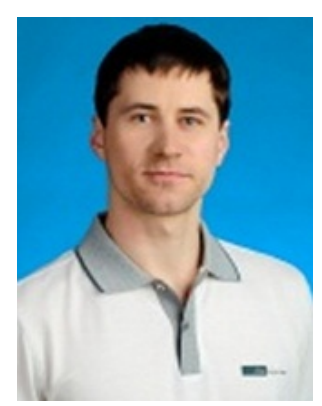

Kaspars Bluss received his BS, MS and Ph.D. degrees from the University of Latvia in 2001, 2002 and 2004 respectively defending his Ph.D. thesis on rate equations for Zeeman coherences for analysis of atoms with radiation. From 2004 to 2007 he was a lecturer at University of Latvia Faculty of Physics and Mathematics. In 2007 he was also elected as senior researcher at University of Latvia Faculty of Physics and Mathematics Physics department. Currently his main work is devoted to development of optical methods for water quality monitoring. These activities are done within the framework of European Social Fund (ESF) project "Formation of interdisciplinary research group for securing the sustainability of salmonid lakes in Latvia"

Address: Šmerḷa 2B, Rīga, LV-1006, Latvia

E-mail: kaspars.bluss@gmail.com 